\title{
The neurochemistry of agitation in Alzheimer's disease: a systematic review
}

Authors:

Kathy Y. Liu ${ }^{1 *}$, Aisling E. Stringer ${ }^{1}$, Suzanne J. Reeves ${ }^{1}$, Robert J. Howard ${ }^{1}$.

Affiliations:

1 Division of Psychiatry, University College London, UK

*corresponding author. Email address kathy.liu@ucl.ac.uk

\section{$\underline{\text { Abstract }}$}

Objective: To provide an up-to-date systematic review of the characteristics, methodology and findings of studies that have investigated the neurochemistry of agitation in Alzheimer's disease (AD).

Methods: Electronic databases were searched for published peer-reviewed articles which provided data on any neurotransmitter system in relation to agitation in AD. Screening of titles and abstracts and data extraction from full texts were conducted in duplicate.

Results: Forty-five studies were included. Monoamines (serotonin, dopamine and noradrenaline) were most commonly investigated. A variety of methods were used to investigate the neurochemistry underlying agitation in $A D$ and, although there were several conflicting findings, there was evidence of serotonergic deficit, relatively preserved dopaminergic function and compensatory overactivity of postsynaptic noradrenergic neurons in agitation in AD. 
Conclusions: Disruption of the dynamic balance between multiple neurotransmitter systems could impair functional neural networks involved in affective regulation and executive function. Differences in study design and methodology may have contributed to conflicting findings. Future studies that overcome these limitations (e.g. using standardized criteria to define agitation) and employ neuroimaging methods such as MRI/PET to investigate specific neural networks are needed to clarify the role of neurotransmitter alterations in these patients.

\section{Keywords:}

agitation; Alzheimer's disease; neurotransmitters; monoamines

\section{Introduction}

Alzheimer's disease (AD) is the most common form of dementia, accounting for up to $60 \%$ of cases (Rizzi et al., 2014) and agitation is a common neuropsychiatric syndrome that affects around $50 \%$ of patients with AD (Aalten et al., 2005; Burns et al., 1990; Lyketsos et al., 2000). Agitation covers a broad range of symptoms including anxiety and irritability, motor restlessness and abnormal vocalization, as well as observable behaviors such as pacing, wandering, aggression, shouting and night-time disturbance (Howard et al., 2001). A recent consensus definition of agitation in dementia characterized the syndrome as observed or inferred evidence of emotional distress associated with excessive motor activity, verbal or physical aggression (Cummings et al., 2015) (Table 1). Agitation significantly reduces quality of life for patients and caregivers, precipitates earlier institutionalisation (Okura et al., 2011), and is associated 
with more rapid disease progression and earlier death (Peters et al., 2015). Although non-pharmacological approaches to the treatment of agitation have shown some evidence of efficacy in a care home setting (Livingston et al., 2014), patients who exhibit more severe agitation with higher levels of distress and associated risk often require additional treatment with medication. However, there are no safe and effective drug treatments for agitation, as the best evidence is for short-term use of atypical antipsychotic drugs, which have modest efficacy and are associated with significant harm (sedation, falls, parkinsonism, stroke) and increased mortality (Ballard and Howard, 2006; Schneider et al., 2006), particularly in those aged over 80 years (Howard et al., 2016). Citalopram, a selective serotonin reuptake inhibitor (SSRI) may also have a degree of efficacy but is associated with worsening cognitive function and cardiac side effects (Porsteinsson et al., 2014).

Table 1: Consensus provisional definition of agitation in cognitive disorders

A. The patient meets criteria for a cognitive impairment or dementia syndrome (e.g. $A D$, FTD, DLB, vascular dementia, other dementias, a pre-dementia cognitive impairment syndrome such as mild cognitive impairment or other cognitive disorder). B. The patient exhibits at least one of the following behaviors that are associated with observed or inferred evidence of emotional distress (e.g. rapid changes in mood, irritability, outbursts). The behavior has been persistent or frequently recurrent for a minimum of two weeks' and represents a change from the patient's usual behavior. (a) Excessive motor activity (examples include: pacing, rocking, gesturing, pointing fingers, restlessness, performing repetitious mannerisms). 
(b) Verbal aggression (e.g. yelling, speaking in an excessively loud voice, using profanity, screaming, shouting).

(c) Physical aggression (e.g. grabbing, shoving, pushing, resisting, hitting others, kicking objects or people, scratching,biting, throwing objects, hitting self, slamming doors, tearing things, and destroying property).

C. Behaviors are severe enough to produce excess disability, which in the clinician's opinion is beyond that due to the cognitive impairment and including at least one of the following:

(a) Significant impairment in interpersonal relationships.

(b) Significant impairment in other aspects of social functioning.

(c) Significant impairment in ability to perform or participate in daily living activities.

D. While co-morbid conditions may be present, the agitation is not attributable solely to another psychiatric disorder, suboptimal care conditions, medical condition, or the physiological effects of a substance.

Disruption of the dynamic balance between cholinergic and monoaminergic neurotransmission has been proposed to underlie the behavioral and psychological symptoms of dementia (BPSD) in AD (Lanari et al., 2006), and previous reviews have summarized the role of specific neurotransmitter systems such as serotonin $(5-\mathrm{HT})$ (Lanctôt et al., 2001) and noradrenaline (NA) (Herrmann et al., 2004b). However, BPSD is a non-specific catch-all that includes a wide range of emotional responses and behaviors (including agitation, apathy, psychosis and depression), which are likely to have distinct neurochemical and neurobiological bases in the AD brain (Lanari et al., 
2006). There is increasing evidence that agitation is caused by dysfunction in specific neuronal networks involved in cognitive control and emotion regulation (Rosenberg et al., 2015), functions known to be modulated by interacting neurotransmitter systems (Del Arco and Mora, 2009). It is therefore logical as well as imperative that we should seek to understand the neurochemical basis of agitation in $A D$ in order to effectively target treatment strategies.

This paper aims to provide an up-to-date systematic review of the characteristics, methodology and findings of studies that have investigated the neurochemistry of agitation in $\mathrm{AD}$. The recently established consensus definition of agitation (Cummings et al., 2015) will be used in the search criteria to optimize cross-study comparison, and the validity of reviewed studies will be discussed.

\section{Methods}

\subsection{Literature search}

Online literature databases (PubMed, PsychINFO, Embase and Web of Science) were searched up to 19th June 2017 using the search terms ("Alzheimer's" OR "Alzheimer" OR "cognitive impairment") AND ("agitation" OR "aggression" OR "restlessness" OR "overactivity" OR "hyperactivity") AND ("neurotransmitter" OR "monoamine" OR "serotonin" OR "dopamine" OR norepinephrine" OR "noradrenaline" OR "acetylcholine" OR "glutamate" OR “GABA").

\subsection{Inclusion/exclusion criteria and screening}


Studies were included if they were published, peer-reviewed papers in human subjects diagnosed with Alzheimer's disease, and provided data on any neurotransmitter system in relation to agitation. Agitated behaviors were defined as excessive motor activity, verbal aggression or physical aggression. Studies were excluded if they did not investigate agitation (as defined above) or describe any method to measure a specific neurotransmitter system. Case reports, poster abstracts and dissertations were also excluded.

\subsection{Data extraction}

Two authors (KL and $A S$ ) independently screened papers for inclusion based on their titles and abstracts, and subsequently extracted data on study characteristics, methods and outcome measures using a structured form. Discrepancies were resolved through discussion.

\section{$\underline{\text { 3. Results }}$}

\subsection{Identification and characteristics of included studies}

Literature searches identified 2269 potential studies, 45 of which met inclusion criteria for data extraction (see Figure 1 PRISMA flow diagram). Most studies investigated one neurotransmitter system which was most commonly serotonin ( $n=17$ studies), followed by dopamine $(n=6)$ and noradrenaline $(n=6)$. Thirteen studies investigated more than one neurotransmitter system. The main characteristics and methodology of these studies are shown in Supplemental Table 1 and are summarized below. 


\subsubsection{Study design and methodology}

Relevant studies included 26 case-control studies, 15 cross-sectional $(n=10)$ or cohort studies $(n=5)$ with no control group and 4 randomized controlled trials. Six out of the 15 studies that did not employ an initial control group divided patients with and without agitation based on subsequent assessment.

The majority of studies employed in vivo methods including genetic analysis $(n=15)$, cerebrospinal fluid (CSF) analysis ( $n=6)$, administration of a neuroendocrine challenge $(n=5)$ or measurement of plasma or platelet markers in blood $(n=4)$. Only one neuroimaging study was included which used positron emission tomography (PET). There were 14 post-mortem studies that measured ex-vivo concentrations of neurotransmitter markers in the brain, of which 6 reported the cause of death and 13 reported the mean duration of post-mortem delay.

A total of 13 different behavioral scales were employed by studies to measure agitation. The most commonly used scale was the Neuropsychiatric Inventory (NPI) ( $n=12)$ followed by the Cohen-Mansfield Agitation Inventory (CMAI) $(n=9)$ and the Behavioral Pathology in Alzheimer's Disease Rating Scale (BEHAVE-AD) $(n=9)$. Other scales that were used included the Neurobehavioral Rating Scale (NBRS), Alzheimer's Disease Assessment Scale (ADAS), Present Behavior Examination (PBE), Brief Psychiatric Rating Scale (BPRS), Manchester and Oxford Universities Scale for the Psychological Assessment of Dementia (MOUSEPAD), retrospective Overt Aggression Scale ( $r-O A S)$ or modified Overt Aggression Scale (OAS-M), Gottfries-Brane-Steen (GBS) scale, 
modified Alzheimer Disease Cooperative Study_Clinical Global Impression of Change (mADCS-CGIC) scale and Behavior Rating scale for Dementia (BRSD). Four studies examined patient clinical records instead of using behavioral scales.

The studies differed in how they temporally defined agitation, which is important as a single measurement could represent a 'state' marker of agitation whereas longitudinal measurements are more likely to detect 'trait' agitation, and these may be underpinned by different neurochemical changes (Lai et al., 2003; Minger et al., 2000). Most ( $n=22)$ studies conducted a single assessment of agitation, whilst others were longitudinal and looked either retrospectively for the occurrence of agitation during the study follow up period ( $n=18)$, or prospectively at several points during the study, such as before and after an intervention $(n=4)$.

\subsubsection{Participants}

Study participants with AD had a mean age of 78 (range 51-97) years and $56 \%$ were female. Twelve studies reported the ethnicity of subjects (mean $58 \%$ White) and two were conducted in non-White Caucasian populations. Studies recruited subjects from the community $(n=4)$, inpatient/care home setting $(n=8)$ or both $(n=7)$. Of 29 studies that provided the mean MMSE score of participants, 15 included subjects with moderate AD (MMSE 10-20) with a mean score of 15 (range 11-19.6), and 14 included subjects with severe $A D(M M S E<10)$ with a mean score of 5 (range 3-8.6). 
Ten studies did not report on the medication status of participants, and of those that did only 5 studies assessed patients who were medication-free. Twenty-four studies did not report the psychiatric history of participants or state whether participants with specific psychiatric conditions were excluded from recruitment.

Five studies included AD subjects who all had agitation, whereas other studies reported a proportion (between $5.6 \%$ to $82 \%$, mean $48 \%$ ) of $A D$ subjects who were defined as agitated. The mean agitation score for these subjects was reported in 19 studies. The mean agitation scores using the three most commonly employed agitation scales were: NPI agitation/aggression subscale (mean 6, range 1.6-8.7), NPI total (31 from one study), CMAI verbal/physical aggression subscale (mean score 14, range 10-20.6), CMAl total (mean 39, range 25-63), BEHAVE-AD aggression subscale (7 from one study), BEHAVE-AD total (mean 10, range 9.2-14.3). Although no official severity threshold scores for these scales are available, the approximate severity of agitation indicated by these mean scores was judged by the authors to be mild to moderate.

\subsection{Main findings}

\subsubsection{Serotonin}

There is a serotonergic deficit in AD (Whitford, 1986), and reductions in 5-HT have been associated with hostility, impulsivity and aggression in other neuropsychiatric patient populations (Duke et al., 2013). Consistent with the hypothesis that serotonergic deficits may be associated with agitation, three studies measured the prolactin response to d,Ifenfluramine (an indirect central 5-HT agonist) and found that aggressive or agitated AD 
patients had higher 5-HT responsivity (Lanctôt et al., 2002a, 2002b; Mintzer et al., 1998)), plausibly due to an overall decline in central 5-HT function with compensatory upregulation of remaining postsynaptic receptors. A further study reported decreased central $5-\mathrm{HT}$ uptake together with lower platelet ${ }^{3} \mathrm{H}$-imipramine binding density in an agitated AD group (Schneider et al., 1988). A subsequent study, however, found no association between aggressive behaviors and platelet $5-\mathrm{HT}$ concentration in AD (Prokšelj et al., 2014).

Measurements of central $5-\mathrm{HT}$ activity in humans have been predominantly based on CSF concentrations of 5-HIAA, the main metabolite of 5-HT (Olivier, 2004). However, reported CSF 5-HIAA concentration in agitated AD patients have been conflicting. Although one study found no correlation with agitation (Engelborghs et al., 2008), others have reported a positive correlation with fear-panic and anxiety scores on the GottfriesBrane-Steen (GBS) scale (Bråne et al., 1989), and higher CSF 5-HIAA concentrations in aggressive compared to both nonaggressive $A D$ patients and healthy older people (Lopez et al., 1996). Similarly, low brain 5-HIAA concentration was found in the hippocampus (Vermeiren et al., 2014a, 2014b), but not in other cortical regions post mortem, in $A D$ patients with agitation or aggression present in their final interview before death ('state' aggression) (Vermeiren et al., 2016).

The serotonergic system is complex, and comprised of multiple serotonergic receptor subtypes, which show region specific differences in their expression and functional properties (Barnes and Sharp, 1999). The evidence for the contribution of specific 
receptors in agitation or aggression appears to be strongest for the $5-\mathrm{HT} 1 \mathrm{~A}$ and $5-\mathrm{HT} 1 \mathrm{~B}$ receptors, as both have been associated with anti-aggressive properties, although underpinning mechanisms have not yet been fully elucidated (Olivier, 2004). 5-HT-1A receptors are abundant in the hippocampus, septum, neocortex and raphe nuclei, and are present on terminals of non-serotonergic neurons, facilitating acetylcholine (ACh), noradrenaline (NA) and dopamine release in the brain, whilst 5-HT1B receptors show high density in the pallidum and substantia nigra and can control the release of GABA, ACh and glutamate neurons (Filip and Bader, 2009). In line with their proposed role in aggression, reviewed studies have shown that $5-\mathrm{HT} 1 \mathrm{~A}$ receptor density is higher in the prefrontal cortex (PFC) (Leake et al., 1993), and lower in the temporal cortex of patients with a history of agitation or aggression during their illness course compared to nonagitated patients (Lai et al., 2003). The PFC has been implicated in behavioral control and aggression as part of a neural circuit also consisting of amygdala, anterior cingulate cortex (ACC) and other interconnected structures, and deficits in this circuit (e.g PFC lesions or hypometabolism) are associated with impulsive aggression in humans (Davidson et al., 2000). In addition, higher 5-HT1A receptor density in frontal cortex, indexed using $\left[{ }^{11} \mathrm{C}\right]$ WAY-10063 PET tracer, was associated with trait aggression in healthy adults (Witte et al., 2009), which the authors attributed to greater post-synaptic 5-HT1A inhibitory activity and PFC hypofunction, leading to reduced control over limbic areas.

So far no specific evidence has implicated a specific contribution of any 5-HT2 receptor subtype to aggression (Olivier, 2004). Similarly, findings in AD patients have been inconclusive: one post-mortem study reported lower cortical 5-HT2 receptor binding in 
aggressive compared to non-aggressive patients (Procter et al., 1992), while another showed no association between 5-HT2 binding in the frontal cortex and severity of agitation (Leake et al., 1993). It is unclear what role other serotonergic receptors might play in agitation (Olivier, 2004). One reviewed study found that patients with higher levels of overactivity, measured using the Present Behavioral Examination (PBE) in the last interview before death, had lower 5-HT6 receptor density in temporal cortex, and that the 5-HT6:ChAT ratio in frontal and temporal cortex correlated with aggression (Garcia-Alloza et al., 2004). The 5-HT6 receptor has been implicated in cognition as it regulates glutamatergic and cholinergic neuronal activity, but its role in agitation has not been established (Woolley et al., 2004), and findings from such studies need confirmation. No studies have investigated the 5-HT3 receptor, which has been implicated in anxiety disorders (Thompson and Lummis, 2007) and thus may be a plausible candidate for contribution to agitation in $A D$. Aside from these receptors, the serotonin transporter (5-HTT) located on serotonergic neurons also plays an important role in the modulation of $5-\mathrm{HT}$ transmission. It has been reported that hippocampal 5HTT sites are preserved or upregulated in physically aggressive AD patients relative to controls (Lai et al., 2011).

Genetic studies have also shown conflicting results. Two studies found a significant association between the 5-HT2A T102C polymorphism and agitation (Assal et al., 2004; Peters et al., 2016), whilst several others, including two performed in populations of non-White ethnicity, did not support this relationship (Holmes et al., 1998; Lai et al., 2011; Lam et al., 2004; Pritchard et al., 2008; Proitsi et al., 2012). Similarly, more 
studies reported no significant association between 5 -HT2C receptor polymorphisms and agitation (Holmes et al., 1998; Pritchard et al., 2008; Proitsi et al., 2012) than one that did (Peters et al., 2016). Genetic analysis of 5-HTT has also provided contradictory findings. A significant association between 5-HTT promoter region (5-HTTPR) polymorphism and aggression was found in two studies (Sukonick et al., 2001; Sweet et al., 2001), and between 5-HTT variable number tandem repeat (VNTR) polymorphism and aggression in another study (Ueki et al., 2007). These relationships, however, were not confirmed in several other studies (Assal et al., 2004; Ha et al., 2005; Peters et al., 2016; Pritchard et al., 2008; Proitsi et al., 2012).

Overall, there is some evidence that serotonergic dysfunction may contribute to agitated behaviors in $A D$ but the exact nature or anatomical location of this dysfunction is unclear. This is partly due to the complexity of the serotonergic system as well as the inconsistent data from studies that have attempted to investigate the precise functional role of the serotoninergic system in this population.

\subsubsection{Dopamine}

There is good evidence that relative preservation of dopaminergic function in the context of diminished cholinergic activity could underlie psychotic symptoms in $A D$ (Cummings et al., 1993), and relative dopaminergic preservation in the context of diminished serotonergic activity in certain brain regions has been linked to aggressive or impulsive behaviors in humans (Seo et al., 2008). Consistent with this hypothesis, reviewed studies have found a negative correlation between ChAT:DA and ChAT:D1 
ratios in temporal cortex and aggressive behavior (Minger et al., 2000), whilst DA concentration alone in post-mortem brain has not been associated with agitation (Bierer et al., 1993; Minger et al., 2000; Vermeiren et al., 2014a, 2014b).

Relative preservation of dopaminergic function in agitated patients is also supported by studies that have examined the concentration of DA metabolites. Preserved concentrations of homovanillic acid (HVA) in the CSF of aggressive patients, relative to healthy controls (Lopez et al., 1996) ${ }_{2}$ and an absence of association between either CSF HVA or 3,4-Dihydroxyphenylacetic acid (DOPAC) and agitation (Bråne et al., 1989; Engelborghs et al., 2008) have been reported. Concentrations of these metabolites in postmortem frontal and temporal cortex was not associated with agitation (Bierer et al., 1993; Minger et al., 2000; Vermeiren et al., 2014a) except in one study which found a correlation between cerebellar DOPAC:DA ratio (indicative of DA turnover) and physical agitation (Vermeiren et al., 2014a). Although a significant relationship was not found between plasma homovanillic acid (HVA) (as a measure of central DA function) and agitation or aggression measured pre- and post-perphenazine administration (Sweet et al., 1997), a trend was found to support a relative preservation of dopaminergic function in agitated patients.

Studies that have performed genetic analyses of DA receptor polymorphisms in agitated AD patients have been mostly negative or inconsistent. Two studies that investigated the relationship between dopamine receptor D1 (DRD1) polymorphism and aggression did not agree on the genotype differences between asymptomatic and symptomatic 
patients, as one reported a greater prevalence of aggression in B2/B2 homozygotes (Sweet et al., 1998), whilst the other did not replicate this and found a stronger association with the B1/B2 genotype (Holmes et al., 2001). A relationship between both DRD1 and dopamine transporter (DAT) VNTR polymorphism and aberrant motor behavior has been reported (Proitsi et al., 2012), but another study found no relationship between DRD1 and agitation (Pritchard et al., 2009). No studies have reported an association between DRD2, DRD3 and DRD4 genetic polymorphisms and agitation (Holmes et al., 2001; Pritchard et al., 2009; Sato et al., 2009; Sweet et al., 1998).

Overall, there is evidence to support the hypothesis that dopaminergic function is relatively preserved in AD patients with agitation. How this system might respond and interact with other neurotransmitter systems in potentially causing such behaviors is still unclear.

\subsubsection{Noradrenaline}

The central noradrenergic system regulates arousal and autonomic function (Samuels and Szabadi, 2008), and there is evidence that hyperactivity of this system is implicated in agitation and aggressive behaviors in humans, including patients with $A D$ (Herrmann et al., 2004b) The locus coeruleus (LC) is the major origin of NA in the CNS and early tau pathology in the LC may precede the hallmark appearance of tau and amyloid pathology in the medial temporal lobe and other cortical regions in AD (Braak et al., 2011)). As $A D$ progresses, the $L C$ experiences loss of neurons and decreases in 
volume (Theofilas et al., 2016). Central NA hyperactivity could result from compensatory activation of remaining noradrenergic LC neurons and/or increased sensitivity of postsynaptic NA receptors.

Several of the reviewed studies reported findings consistent with this hypothesis. Using the highest aggression scores recorded during the course of the patients' illness, a postmortem analysis found greater loss of neurons from the rostral LC in aggressive compared to non-aggressive AD patients (Matthews et al., 2002). Patients with moderate AD (mean MMSE score 14) showed significantly greater agitation in response to yohimbine (an $\alpha 2$-adrenergic antagonist which stimulates NA activity by blocking NAmediated feedback inhibition of LC neurons) compared to age-matched healthy controls, and this correlated with higher CSF NA concentration (Peskind et al., 1998). However the association between CSF NA concentration and agitation did not reach significance in another study (Engelborghs et al., 2008).

Accumulating evidence supports an inverted-U dose-response model of noradrenergic function which proposes that states of low stress are associated with moderate tonic LC neuron discharge resulting in enhanced PFC function via high-affinity a2-adrenoceptor activation, whereas states of high stress are associated with elevated LC discharge and binding to low-affinity a1-adrenoceptors resulting in impaired PFC function (Arnsten, 2009). It is therefore plausible that the compensatory central overactivity of NA in response to LC neuron loss in AD could shift NA actions in the PFC to the right hand side of the curve, resulting in impaired attention, disinhibition and affective 
dysregulation. Consistent with this model, aggressive AD patients showed higher postmortem a1-adrenoceptor density and affinity in the frontal cortex (Sharp et al., 2007) and a blunted growth hormone response to a clonidine challenge (an $\alpha 2$-adrenergic receptor agonist) which was interpreted to reflect compensatory down-regulation of postsynaptic central a2-adrenoceptors in response to enhanced NA outflow (Herrmann et al., 2004a).

In the studies reviewed, ex-vivo measurements of brain noradrenaline (NA) and adrenoceptor concentrations showed region-specific variation. No significant differences in frontal and temporal cortical NA concentration were found between aggressive and nonaggressive AD patients (Matthews et al., 2002; Vermeiren et al., 2016, 2014a), however the hippocampus had a lower NA concentration in aggressive patients (Vermeiren et al., 2014b). Aggressive AD subjects had higher $\alpha 2, \beta 1$ and $\beta 2-$ adrenoceptor levels in the cerebellum (Russo-Neustadt and Cotman, 1997) but not in frontal, temporal or thalamic regions (Matthews et al., 2002; Russo-Neustadt and Cotman, 1997).

Neuropeptide $\mathrm{Y}$ (NPY), a neurotransmitter believed to play a role in anxiety, reduces noradrenergic activity (Illes and Regenold, 1990), so low NPY could enhance the noradrenergic contribution to agitation. Indeed, restlessness and agitated behavior in AD was negatively correlated with CSF levels NPY(Minthon et al., 1996), although CSF NPY was also significantly correlated with duration of illness, so level of cognitive impairment could have been a confounding factor. 
Overall, there is some evidence to support the hypothesis that aggressive behaviors in $A D$ may result from compensatory overactivity of the NA system, secondary to neuronal loss in the LC with preserved or increased postsynaptic a1-adrenoceptors in a variety of brain regions. These regions (e.g. frontal cortex) are part of a functional network involved in cognitive control and emotional regulation.

\subsubsection{Other neurotransmitter systems: acetylcholine and GABA}

AD associated cholinergic deficits have been associated with both cognitive changes (Coyle et al., 1983) and BPSD (Cummings and Kaufer, 1996). Lower concentrations of choline acetyltransferase (ChAT) and/or acetylcholine (ACh) in temporal and frontal cortex were associated with aggressive behavior (Garcia-Alloza et al., 2005) and overactivity (Minger et al., 2000), however this has not been consistently shown (Leake et al., 1993; Procter et al., 1992), and more advanced disease stage (indexed by lower MMSE scores) in those with more marked cholinergic deficiency is a potential confounding factor. There was evidence that disruption of the dynamic balance between cholinergic activity and other neurotransmitter systems could make individuals with $A D$ more susceptible to agitated behaviors. For example, the ChAT:DA and ChAT:D1 ratios in frontal and temporal cortex were negatively correlated with overactivity and aggressive behavior respectively in AD (Minger et al., 2000), and the 5-HT6:ChAT ratio in frontal and temporal cortex was also related to aggression (Garcia-Alloza et al., 2004). In addition, a PET study in agitated subjects with AD found lower $\alpha 4 \beta 2^{*}$ nicotinic cholinergic receptor binding in bilateral anterior cingulate cortex (ACC) (Sultzer et al., 
2017)). Nicotinic cholinergic receptors play an important role in influencing the release of other neurotransmitters (Dani, 2001), thus reduced receptor availability in a region that modulates behavioral and emotional activation could contribute to agitation via the postsynaptic activity of other neurotransmitter systems.

$\gamma$-aminobutyric acid (GABA) is the key inhibitory neurotransmitter of the central nervous system and GABAergic dysfunction in AD has been proposed to contribute to BPSD (Lanctôt et al., 2004). There are numerous $\mathrm{GABA}_{\mathrm{A}}$ receptor subtypes, each with distinct properties owing to the different possible combinations of receptor subunits (Chebib and Johnston, 1999) and the subunit composition can affect receptor sensitivity to modulators such as benzodiazepines and alcohol (Rudolph and Möhler, 2004), resulting in either increased or decreased aggression (de Almeida et al., 2005). The two studies that investigated $\gamma$-aminobutyric acid (GABA) included in this review found no significant relationship between either plasma GABA (Lanctôt et al., 2007) or cortical GABA concentrations (Procter et al., 1992) and aggression in AD, and did not investigate specific GABA receptor subtypes.

Overall, apart from 5-HT, dopamine and NA, a small number of studies have implicated other neurotransmitter systems in agitation but these need further replication to make any conclusions about their possible role in agitation in AD.

\section{$\underline{\text { 4. Discussion }}$}


The reviewed studies used a variety of methods to investigate the neurochemistry underlying agitation in Alzheimer's disease. Although some conclusions can be drawn about the potential role of specific neurotransmitter systems in agitation in $A D$, there were several conflicting findings. Here we discuss the findings and the degree to which some of the differences in study design and methodology may have influenced the consistency and validity of results and the ability to compare across studies. A number of recommendations for future research will also be described.

Agitation in dementia has recently been defined as a syndrome characterized by inferred or observed evidence of emotional distress associated with at least one behavioral component, i.e. excessive motor activity, verbal or physical aggression (Cummings et al., 2015). Subjective distress is a core feature due to the consensus that agitated behavior is an expression of the person's distressed emotional state, which can manifest as rapid changes in mood, irritability or emotional outbursts. This points to potential deficits in emotion regulation in agitated individuals with $A D$. A recent review (Rosenberg et al., 2015) found neuroimaging evidence of dysfunction in what could be termed 'agitation circuits' in AD subjects, comprising frontal cortex, ACC, orbitofrontal cortex, amygdala and insula, regions also implicated in anxiety (Grupe and Nitschke, 2013). Neuroimaging studies of emotion regulation have revealed that subcortical emotion structures (especially the amygdala) are under direct regulatory control of the PFC (Ochsner et al., 2004), and frontal lobe or fronto-subcortical dysfunction results in greater disinhibition and reduced regulation of emotion and aggressive behaviors (Seo et al., 2008). From a neuropsychological perspective, agitation in AD could arise from a 
tendency to misinterpret/overestimate potential threats and respond with heightened emotional reactivity. This provides a testable hypothesis that can potentially be translated to human studies, although the applicability of such neuropsychological paradigms (which have usually been developed in healthy young adults) to patients with $A D$, especially in those with severe cognitive impairment, needs further study. In terms of the underlying neurobiological mechanisms, the prevalence of agitation increases with disease severity (Lyketsos et al., 2000), which suggests an association between the neurochemical processes accompanying $A D$ disease progression and agitation.

Nonetheless, the precise nature and combined effect of multiple neurochemical imbalances have not yet been fully elucidated. Surprisingly few studies have focused on brain regions proposed to be part of the 'agitation circuit' in $A D$ subjects comprising frontal cortex, ACC, orbitofrontal cortex, amygdala and insula. Additional gaps identified in the research literature include the potential roles of $5-\mathrm{HT} 3$ receptor and $\mathrm{GABA}_{A}$ receptor subtypes. There were numerous conflicting findings especially within genetic studies, and many studies would benefit from replication. The main methodological issues of studies are addressed below.

\subsection{Limitations of reviewed studies}

Genetic analysis was the most commonly employed method within the studies that we reviewed, however several genetic studies recruited fewer than one hundred subjects in each group (Assal et al., 2004; Ha et al., 2005; Lam et al., 2004; Sukonick et al., 2001). These were probably underpowered to detect any significant associations as a very 
large sample size of many hundreds of subjects is usually required to detect a potentially small effect size of a genetic polymorphism (Hong and Park, 2012). Several genetic studies have also investigated the influence of a number of different polymorphisms relating to various neurotransmitter systems, but few reported making any statistical correction for multiple comparisons, which in one study made their apparent result non-significant (Pritchard et al., 2009). In addition, a number of in vivo studies measured peripheral concentrations of neurotransmitters or their metabolites to investigate brain neurotransmitter activity, but the specificity of these methods has not been established.

Medication status and psychiatric history of subjects were incompletely reported, which could have influenced the neurochemical findings of in-vivo studies and the detection of agitation due to treatment-induced reduction of frequency/severity of agitated behavior. Although medications may target specific receptors or neurotransmitter systems, (e.g. cholinesterase inhibitors which enhance the cholinergic system) they may also indirectly modulate the neurotransmission of other systems (e.g. presynaptic nicotinic acetylcholine receptors are found on serotonergic, dopaminergic and noradrenergic neurons in the brain (Wonnacott, 1997)). Although a few studies reported post-hoc the absence of a statistical effect from medication or psychiatric history, it has been reported that chronic use of psychotropic medication could upregulate adrenergic receptors in frontal cortex in agitated AD patients (Sharp et al., 2007), and more widely it has been shown using PET that chronic antipsychotic administration can alter brain 
neurochemistry in patients with schizophrenia by increasing striatal dopamine receptors (Silvestri et al., 2000).

The consensus definition of agitation applies to a chronic state that has been sustained or been frequently recurrent for at least 2 weeks (Cummings et al., 2015). Thus a single 'snapshot' measurement of agitation, which was conducted in 22 studies, is likely to be less accurate than multiple retrospective/prospective measurements in detecting agitation in $A D$, unless it has included an assessment of behavior during the preceding 2 weeks. This is relevant because 'state' versus 'trait' agitation may have distinct etiologies and be differentially regulated by neurotransmitter systems (Olivier, 2004). Although agitation in AD may persist for years, it can be episodic and the severity may fluctuate (van der Linde et al., 2016), so that studies that have assessed patients with $\mathrm{mild} / \mathrm{moderate} A D$ may not have detected the 'true' final prevalence of agitation which has been shown to increase with worsening disease progression and severity of cognitive impairment (Lyketsos et al., 2000).

Another factor that may have contributed to discrepancies between studies is how agitated behaviors have been classified. The consensus definition of agitation requires that there be observed or inferred evidence of emotional distress (Cummings et al., 2015), however, none of the reviewed studies were judged to have adequately reported this. Aggression was the most commonly measured behavior and was investigated exclusively in 15 studies. While agitation may include aggressive behaviors, agitation can also occur without aggression (e.g. pacing, rocking, restlessness). These studies 
did not measure other agitated behaviors such as motor overactivity without aggression, which may have influenced their findings. Agitated behaviors were measured using a variety of different rating scales which are difficult to aggregate or compare across studies as most do not have established severity thresholds. For example, it is not possible to distinguish from the NPI and CMAI how the frequency or severity of behaviors contributed to the total score. The approximate severity of agitation indicated by the mean scores of the three most widely used scales (NPI, CMAI, BEHAVE-AD) were judged by the authors to be mild to moderate, therefore the results of this review may not be generalizable to individuals with severe agitation.

To our knowledge, only one neuroimaging study (using PET) has investigated the neurochemistry of agitation in $A D$, perhaps due to the obvious challenges of recruiting and scanning this population. However, this and a previous study which used PET in older adults with delusions (Reeves et al., 2009) (a symptom frequently associated with agitation (Gilley et al., 1997)), demonstrates the feasibility of employing neuroimaging techniques in this population. Radiolabelled agonists or antagonists used to investigate the altered binding potential of specific receptor subtypes in vivo may provide additional support for the regional neurotransmitter deficits reported in post-mortem studies. Relevant radioligands are available for the dopaminergic (e.g. DRD1) and serotonergic (e.g. 5-HT1A) receptors, but there is a lack of relevant radioligands for the noradrenergic system. Although magnetic resonance imaging (MRI) cannot be used to visualize neuroreceptor binding, the integrity of the LC and substantia nigra (major sources of NA and dopamine neurons respectively) can be measured using T1- or 
magnetization transfer (MT)-weighted approaches (Nakane et al., 2008), also known as 'neuromelanin-sensitive' MRI. Despite the challenges of imaging a small structure such as the LC, previous studies have consistently reported differences in LC signal intensity between patients with AD and healthy controls (Liu et al., 2017). However, more studies are needed to confirm the validity of using LC signal intensity as a potential biomarker of NA system integrity in $A D$.

\subsection{Recommendations for future studies}

To optimize the ability to aggregate and compare findings across studies, future studies should try to classify agitation in the same way, e.g. by using the recent consensus definition of agitation (Cummings et al., 2015). Further work is needed to investigate the neurochemistry of different components of agitation including behaviors indicative of emotional distress. Ideally, studies should use standard agitation scales such as the CMAI and NPI and report the breakdown of scores, i.e. frequency and severity subscores for agitated behaviors. Longitudinal assessments may be better than crosssectional measurements to detect agitated behaviors in mild/moderate $A D$, as the prevalence of agitation increases with $A D$ severity.

To improve the validity of findings, studies should be adequately powered and should try to account for medication and other psychiatric conditions, e.g. by including an agematched control group if possible, as these may contribute to neurotransmitter alterations. In post-mortem studies, the post-mortem delay, brain $\mathrm{pH}$ and cause of death 
are additional important parameters, as the ability to interpret postmortem studies is limited by agonal events as well as perimortem medication use.

Further studies are needed to support and refine the hypotheses regarding the neurochemistry underlying agitation in $A D$ outlined in this review. The evolution of neurochemical changes and the interaction of different neurotransmitter systems also require clarification. Neuroimaging techniques, e.g. PET-MRI technology, can be employed for simultaneous investigation of functional connectivity to explore specific neural networks, and PET neuroreceptor imaging (e.g. DRD1, 5-HT1A receptors) in specific brain regions including frontal cortex, ACC, orbitofrontal cortex, amygdala and insula, as these have been proposed to form part of an 'agitation circuit' within which PFC regulatory control of subcortical emotional centers may be dysfunctional. However, in the later stages of $A D$ when agitation becomes more prevalent, the extent of neuron and synapse loss may make interrogation of specific brain circuits difficult to interpret and possibly uninformative. The use of 'neuromelanin-sensitive' MRI techniques to measure LC or substantia nigra signal intensity can potentially assess the integrity of the noradrenergic or dopaminergic system respectively in AD. However, the difficulty associated with scanning agitated individuals with $A D$ is likely to increase with illness severity and degree of agitation, and severely agitated individuals may not be able to tolerate the procedure without sedation.

Pharmacological studies of agitation in $A D$ that use medication to target a specific receptor system may also provide insight into the aetiology of agitation and effective 
treatment strategies. For example, agents that improve functional serotonin neurotransmission such as citalopram, a selective serotonin reuptake inhibitor (SSRI), and carbamazepine, a sodium channel channel blocker and serotonin releasing agent, have been shown to be superior to placebo in treating agitation and aggression in $A D$ (Porsteinsson et al., 2014; Tariot et al., 1998). Prazosin, an a1-blocker, led to greater improvement in $\mathrm{AD}$ patients with treatment-resistant agitation compared to placebo (Wang et al., 2009), supporting the proposed role of enhanced responsiveness to NA at the a1-adrenoreceptor in dementia-related agitation. Interestingly, although cholinesterase inhibitors are well tolerated, their main benefits for BPSD are probably for anxiety and apathy rather than agitation (Ballard et al., 2009). A recent clinical trial also implicated the cannabinoid system in AD-related agitation (Woodward et al., 2014)). Given the evidence for the likely involvement of multiple neurotransmitter systems in AD-related agitation, an optimal drug therapy may be one that targets multiple receptors from different neurotransmitter systems. However, this may also lead to increased side effects and greater difficulty investigating the role of specific neurotransmitter systems. For example, risperidone acts as an antagonist or inverse agonist at multiple receptors belonging to the dopaminergic, serotonergic and noradrenergic systems. Memantine has shown some benefit in clinical trials and targets the glutaminergic system (NMDA receptor), but also acts as an antagonist to serotonergic $\left(5-\mathrm{HT}_{3}\right)$ and cholinergic (nicotinic $\mathrm{ACh}$ ) receptors and an agonist at dopaminergic (DRD2) receptors. Further clinical trials are required to determine the efficacy, safety and tolerability of potential pharmacological treatments, whilst 
elucidating the contribution/interaction of different neurotransmitter systems that may underlie agitation.

\subsection{Limitations of the review}

This review concentrated on neurotransmitter system changes in agitation and did not include studies that investigated only structural (e.g. regional brain volume) or functional changes (e.g. cerebral blood flow or glucose metabolism). Neither were treatment studies included if they did not specifically report methods to measure a neurotransmitter system. Although inclusion of these studies may have provided additional information to support the involvement of specific neurotransmitter systems and/or functional networks, this data would have been less specific. This review also did not include agitated patients who had other neuropsychiatric conditions such as other dementias or psychotic disorders, which may have provided further insight into the neurochemistry of agitation, although this would have limited the ability to crosscompare studies. The recent (2015) consensus definition of agitation that guided our search criteria emerged after the publication of most reviewed studies, so it was not clear if all agitated behaviors were associated with behaviors indicative of emotional distress as this was not typically reported or measured. A quantitative statistical review was not the aim of this study so it cannot comment on effect sizes.

\section{$\underline{5 . \text { Conclusion }}$}

This review has found evidence to support the presence of serotonergic dysfunction, relatively preserved dopaminergic function and compensatory overactivity of the NA 
system in agitation in AD. These could impair functional neural networks involved in affective regulation and executive function. However, a causal relationship cannot be derived from these studies, and differences in study design and methodology may contribute to conflicting findings and a limited ability to cross-compare results. Future studies that overcome these limitations and employ standardized neuroimaging techniques methods such MRI and PET across the AD course are needed to clarify the role of neurotransmitter alterations and target appropriate drug treatments for agitation.

\section{Acknowledgements}

We are grateful to the anonymous reviewer for their helpful comments. The views expressed are those of the authors and not necessarily those of the NHS or the Department of Health. $\mathrm{RH}$ is supported by the UCLH NIHR BRC. Declarations of interest: none.

\section{$\underline{\text { References: }}$}

Aalten, P., de Vugt, M.E., Jaspers, N., Jolles, J., Verhey, F.R.J., 2005. findings from the two-year longitudinal Maasbed study. Int. J. Geriatr. Psychiatry 20, 523-530.

Arnsten, A.F.T., 2009. Stress signalling pathways that impair prefrontal cortex structure and function. Nat. Rev. Neurosci. 10, 410-422.

Assal, F., Alarcón, M., Solomon, E.C., Masterman, D., Geschwind, D.H., Cummings, J.L., 2004. Association of the serotonin transporter and receptor gene polymorphisms in neuropsychiatric symptoms in Alzheimer disease. Arch. Neurol. 61, 1249-1253.

Ballard, C.G., Gauthier, S., Cummings, J.L., Brodaty, H., Grossberg, G.T., Robert, P., Lyketsos, C.G., 2009. Management of agitation and aggression associated with Alzheimer disease. Nat. Rev. Neurol. 5, 245-255.

Ballard, C., Howard, R., 2006. Neuroleptic drugs in dementia: benefits and harm. Nat. Rev. Neurosci. 7, 492-500.

Barnes, N.M., Sharp, T., 1999. A review of central 5-HT receptors and their function. Neuropharmacology 38, 1083-1152.

Bierer, L.M., Knott, P.J., Schmeidler, J.M., Marin, D.B., Ryan, T.M., Haroutunian, V., 
Purohit, D.P., Perl, D.P., Mohs, R.C., Davis, K.L., 1993. Post-mortem examination of dopaminergic parameters in Alzheimer's disease: Relationship to noncognitive symptoms. Psychiatry Res. 49, 211-217.

Braak, H., Thal, D.R., Ghebremedhin, E., Del Tredici, K., 2011. Stages of the pathologic process in Alzheimer disease: age categories from 1 to 100 years. J. Neuropathol. Exp. Neurol. 70, 960-969.

Bråne, G., Gottfries, C.G., Blennow, K., Karlsson, I., Lekman, A., Parnetti, L., Svennerholm, L., Wallin, A., 1989. Monoamine metabolites in cerebrospinal fluid and behavioral ratings in patients with early and late onset of Alzheimer dementia. Alzheimer Dis. Assoc. Disord. 3, 148-156.

Burns, A., Jacoby, R., Levy, R., 1990. Psychiatric phenomena in Alzheimer's disease. IV: Disorders of behaviour. Br. J. Psychiatry 157, 86-94.

Chebib, M., Johnston, G.A.R., 1999. THE "ABC" OF GABA RECEPTORS: A BRIEF REVIEW. Clinical and Experimental Pharmacology and Physiology 26, 937-940.

Coyle, J.T., Price, D.L., DeLong, M.R., 1983. Alzheimer's disease: a disorder of cortical cholinergic innervation. Science 219, 1184-1190.

Cummings, J.L., Gorman, D.G., Shapira, J., 1993. Physostigmine ameliorates the delusions of Alzheimer's disease. Biol. Psychiatry 33, 536-541.

Cummings, J.L., Kaufer, D., 1996. Neuropsychiatric aspects of Alzheimer's disease: the cholinergic hypothesis revisited. Neurology 47, 876-883.

Cummings, J., Mintzer, J., Brodaty, H., Sano, M., Banerjee, S., Devanand, D.P., Gauthier, S., Howard, R., Lanctôt, K., Lyketsos, C.G., Peskind, E., Porsteinsson, A.P., Reich, E., Sampaio, C., Steffens, D., Wortmann, M., Zhong, K., International Psychogeriatric Association, 2015. Agitation in cognitive disorders: International Psychogeriatric Association provisional consensus clinical and research definition. Int. Psychogeriatr. 27, 7-17.

Dani, J.A., 2001. Overview of nicotinic receptors and their roles in the central nervous system. Biol. Psychiatry 49, 166-174.

Davidson, R.J., Putnam, K.M., Larson, C.L., 2000. Dysfunction in the neural circuitry of emotion regulation--a possible prelude to violence. Science 289, 591-594.

de Almeida, R.M.M., Ferrari, P.F., Parmigiani, S., Miczek, K.A., 2005. Escalated aggressive behavior: dopamine, serotonin and GABA. Eur. J. Pharmacol. 526, 5164.

Del Arco, A., Mora, F., 2009. Neurotransmitters and prefrontal cortex-limbic system interactions: implications for plasticity and psychiatric disorders. J. Neural Transm. 116, 941-952.

Duke, A.A., Bègue, L., Bell, R., Eisenlohr-Moul, T., 2013. Revisiting the serotoninaggression relation in humans: a meta-analysis. Psychol. Bull. 139, 1148-1172.

Engelborghs, S., Vloeberghs, E., Le Bastard, N., Van Buggenhout, M., Mariën, P., Somers, N., Nagels, G., Pickut, B.A., De Deyn, P.P., 2008. The dopaminergic neurotransmitter system is associated with aggression and agitation in frontotemporal dementia. Neurochem. Int. 52, 1052-1060.

Filip, M., Bader, M., 2009. Overview on 5-HT receptors and their role in physiology and pathology of the central nervous system. Pharmacol. Rep. 61, 761-777.

Garcia-Alloza, M., Gil-Bea, F.J., Diez-Ariza, M., Chen, C.P.L.-H., Francis, P.T., Lasheras, B., Ramirez, M.J., 2005. Cholinergic-serotonergic imbalance contributes 
to cognitive and behavioral symptoms in Alzheimer's disease. Neuropsychologia 43, 442-449.

Garcia-Alloza, M., Hirst, W.D., Chen, C.P.L.-H., Lasheras, B., Francis, P.T., Ramírez, M.J., 2004. Differential involvement of 5-HT(1B/1D) and 5-HT6 receptors in cognitive and non-cognitive symptoms in Alzheimer's disease.

Neuropsychopharmacology 29, 410-416.

Gilley, D.W., Wilson, R.S., Beckett, L.A., Evans, D.A., 1997. Psychotic symptoms and physically aggressive behavior in Alzheimer's disease. J. Am. Geriatr. Soc. 45, 1074-1079.

Grupe, D.W., Nitschke, J.B., 2013. Uncertainty and anticipation in anxiety: an integrated neurobiological and psychological perspective. Nat. Rev. Neurosci. 14, 488-501.

Ha, T.M., Cho, D.M., Park, S.W., Joo, M.J., Lee, B.J., Kong, B.G., Kim, J.M., Yoon, J.S., Kim, Y.H., 2005. Evaluating associations between 5-HTTLPR polymorphism and Alzheimer's disease for Korean patients. Dement. Geriatr. Cogn. Disord. 20, 31-34.

Herrmann, N., Lanctôt, K.L., Eryavec, G., Van Reekum, R., Khan, L.R., 2004a. Growth hormone response to clonidine predicts aggression in Alzheimer's disease.

Psychoneuroendocrinology 29, 1192-1197.

Herrmann, N., Lanctôt, K.L., Khan, L.R., 2004b. The role of norepinephrine in the behavioral and psychological symptoms of dementia. J. Neuropsychiatry Clin. Neurosci. 16, 261-276.

Holmes, C., Arranz, M.J., Powell, J.F., Collier, D.A., Lovestone, S., 1998. 5-HT2A and 5-HT2C receptor polymorphisms and psychopathology in late onset Alzheimer's disease. Hum. Mol. Genet. 7, 1507-1509.

Holmes, C., Smith, H., Ganderton, R., Arranz, M., Collier, D., Powell, J., Lovestone, S., 2001. Psychosis and aggression in Alzheimer's disease: the effect of dopamine receptor gene variation. J. Neurol. Neurosurg. Psychiatry 71, 777-779.

Hong, E.P., Park, J.W., 2012. Sample size and statistical power calculation in genetic association studies. Genomics Inform. 10, 117-122.

Howard, R., Ballard, C., O'Brien, J., Burns, A., UK and Ireland Group for Optimization of Management in dementia, 2001. Guidelines for the management of agitation in dementia. Int. J. Geriatr. Psychiatry 16, 714-717.

Howard, R., Costafreda, S.G., Karcher, K., Coppola, D., Berlin, J.A., Hough, D., 2016. Baseline characteristics and treatment-emergent risk factors associated with cerebrovascular event and death with risperidone in dementia patients. $\mathrm{Br}$. J. Psychiatry 209, 378-384.

Illes, P., Regenold, J.T., 1990. Interaction between neuropeptide $Y$ and noradrenaline on central catecholamine neurons. Nature 344, 62-63.

Lai, M.K.P., Tsang, S.W., Esiri, M.M., Francis, P.T., Wong, P.T.-H., Chen, C.P., 2011. Differential involvement of hippocampal serotonin1A receptors and re-uptake sites in non-cognitive behaviors of Alzheimer's disease. Psychopharmacology 213, 431439.

Lai, M.K.P., Tsang, S.W.Y., Francis, P.T., Esiri, M.M., Keene, J., Hope, T., Chen, C.P.L.-H., 2003. Reduced serotonin 5-HT1A receptor binding in the temporal cortex correlates with aggressive behavior in Alzheimer disease. Brain Res. 974, 82-87.

Lam, L.C.W., Tang, N.L.S., Ma, S.L., Zhang, W., Chiu, H.F.K., 2004. 5-HT2A T102C receptor polymorphism and neuropsychiatric symptoms in Alzheimer's disease. Int. 
J. Geriatr. Psychiatry 19, 523-526.

Lanari, A., Amenta, F., Silvestrelli, G., Tomassoni, D., Parnetti, L., 2006.

Neurotransmitter deficits in behavioural and psychological symptoms of Alzheimer's disease. Mech. Ageing Dev. 127, 158-165.

Lanctôt, K.L., Herrmann, N., Eryavec, G., van Reekum, R., Reed, K., Naranjo, C.A., 2002a. Central serotonergic activity is related to the aggressive behaviors of Alzheimer's disease. Neuropsychopharmacology 27, 646-654.

Lanctôt, K.L., Herrmann, N., Mazzotta, P., 2001. Role of serotonin in the behavioral and psychological symptoms of dementia. J. Neuropsychiatry Clin. Neurosci. 13, 5-21.

Lanctôt, K.L., Herrmann, N., Mazzotta, P., Khan, L.R., Ingber, N., 2004. GABAergic function in Alzheimer's disease: evidence for dysfunction and potential as a therapeutic target for the treatment of behavioural and psychological symptoms of dementia. Can. J. Psychiatry 49, 439-453.

Lanctôt, K.L., Herrmann, N., Rothenburg, L., Eryavec, G., 2007. Behavioral correlates of GABAergic disruption in Alzheimer's disease. Int. Psychogeriatr. 19, 151-158.

Lanctôt, K.L., Herrmann, N., van Reekum, R., Eryavec, G., Naranjo, C.A., 2002b. Gender, aggression and serotonergic function are associated with response to sertraline for behavioral disturbances in Alzheimer's disease. Int. J. Geriatr. Psychiatry $17,531-541$.

Leake, A., Moore, P.B., Leitch, M., Ayre, K., Perry, R.H., Ince, P.G., Ferrier, I.N., 1993. The serotonergic system in Alzheimer's disease and normal neocortical postmortem brain: neurochemical and clinical correlates. Neurol Psychiatry Brain Res 2, 53-59.

Liu, K.Y., Marijatta, F., Hämmerer, D., Acosta-Cabronero, J., Düzel, E., Howard, R.J., 2017. Magnetic resonance imaging of the human locus coeruleus: A systematic review. Neurosci. Biobehav. Rev. 83, 325-355.

Livingston, G., Kelly, L., Lewis-Holmes, E., Baio, G., Morris, S., Patel, N., Omar, R.Z., Katona, C., Cooper, C., 2014. Non-pharmacological interventions for agitation in dementia: systematic review of randomised controlled trials. Br. J. Psychiatry 205, 436-442.

Lopez, O.L., Kaufer, D., Reiter, C.T., Carra, J., DeKosky, S.T., Palmer, A.M., 1996. Relationship between CSF neurotransmitter metabolites and aggressive behavior in Alzheimer's disease. Eur. J. Neurol. 3, 153-155.

Lyketsos, C.G., Steinberg, M., Tschanz, J.T., Norton, M.C., Steffens, D.C., Breitner, J.C., 2000. Mental and behavioral disturbances in dementia: findings from the Cache County Study on Memory in Aging. Am. J. Psychiatry 157, 708-714.

Matthews, K.L., Chen, C.P.L.-H., Esiri, M.M., Keene, J., Minger, S.L., Francis, P.T., 2002. Noradrenergic changes, aggressive behavior, and cognition in patients with dementia. Biol. Psychiatry 51, 407-416.

Minger, S.L., Esiri, M.M., McDonald, B., Keene, J., Carter, J., Hope, T., Francis, P.T., 2000. Cholinergic deficits contribute to behavioral disturbance in patients with dementia. Neurology 55, 1460-1467.

Minthon, L., Edvinsson, L., Gustafson, L., 1996. Correlation between clinical characteristics and cerebrospinal fluid neuropeptide $Y$ levels in dementia of the Alzheimer type and frontotemporal dementia. Alzheimer Dis. Assoc. Disord. 10, 197-203. 
Mintzer, J., Brawman-Mintzer, O., Mirski, D.F., Unger, R., Nietert, P., Meeks, A., Sampson, R., 1998. Fenfluramine challenge test as a marker of serotonin activity in patients with Alzheimer's dementia and agitation. Biol. Psychiatry 44, 918-921.

Nakane, T., Nihashi, T., Kawai, H., Naganawa, S., 2008. Visualization of neuromelanin in the Substantia nigra and locus ceruleus at 1.5T using a 3D-gradient echo sequence with magnetization transfer contrast. Magn. Reson. Med. Sci. 7, 205210.

Ochsner, K.N., Ray, R.D., Cooper, J.C., Robertson, E.R., Chopra, S., Gabrieli, J.D.E., Gross, J.J., 2004. For better or for worse: neural systems supporting the cognitive down- and up-regulation of negative emotion. Neuroimage 23, 483-499.

Okura, T., Plassman, B.L., Steffens, D.C., Llewellyn, D.J., Potter, G.G., Langa, K.M., 2011. Neuropsychiatric symptoms and the risk of institutionalization and death: the aging, demographics, and memory study. J. Am. Geriatr. Soc. 59, 473-481.

Olivier, B., 2004. Serotonin and aggression. Ann. N. Y. Acad. Sci. 1036, 382-392.

Peskind, E.R., Elrod, R., Dobie, D.J., Pascualy, M., Petrie, E., Jensen, C., Brodkin, K., Murray, S., Veith, R.C., Raskind, M.A., 1998. Cerebrospinal fluid epinephrine in Alzheimer's disease and normal aging. Neuropsychopharmacology 19, 465-471.

Peters, M.E., Schwartz, S., Han, D., Rabins, P.V., Steinberg, M., Tschanz, J.T., Lyketsos, C.G., 2015. Neuropsychiatric symptoms as predictors of progression to severe Alzheimer's dementia and death: the Cache County Dementia Progression Study. Am. J. Psychiatry 172, 460-465.

Peters, M.E., Vaidya, V., Drye, L.T., Devanand, D.P., Mintzer, J.E., Pollock, B.G., Porsteinsson, A.P., Rosenberg, P.B., Schneider, L.S., Shade, D.M., Weintraub, D., Yesavage, J., Lyketsos, C.G., Avramopoulos, D., CitAD Research Group, 2016. Citalopram for the Treatment of Agitation in Alzheimer Dementia: Genetic Influences. J. Geriatr. Psychiatry Neurol. 29, 59-64.

Porsteinsson, A.P., Drye, L.T., Pollock, B.G., Devanand, D.P., Frangakis, C., Ismail, Z., Marano, C., Meinert, C.L., Mintzer, J.E., Munro, C.A., Pelton, G., Rabins, P.V., Rosenberg, P.B., Schneider, L.S., Shade, D.M., Weintraub, D., Yesavage, J., Lyketsos, C.G., CitAD Research Group, 2014. Effect of citalopram on agitation in Alzheimer disease: the CitAD randomized clinical trial. JAMA 311, 682-691.

Pritchard, A.L., Harris, J., Pritchard, C.W., Coates, J., Haque, S., Holder, R., Bentham, P., Lendon, C.L., 2008. Role of 5HT2A and 5HT2C polymorphisms in behavioural and psychological symptoms of Alzheimer's disease. Neurobiol. Aging 29, 341347.

Pritchard, A.L., Ratcliffe, L., Sorour, E., Haque, S., Holder, R., Bentham, P., Lendon, C.L., 2009. Investigation of dopamine receptors in susceptibility to behavioural and psychological symptoms in Alzheimer's disease. Int. J. Geriatr. Psychiatry 24, 1020-1025.

Procter, A.W., Francis, P.T., Stratmann, G.C., Bowen, D.M., 1992. Serotonergic pathology is not widespread in Alzheimer patients without prominent aggressive symptoms. Neurochem. Res. 17, 917-922.

Proitsi, P., Lupton, M.K., Reeves, S.J., Hamilton, G., Archer, N., Martin, B.M., lyegbe, C., Hollingworth, P., Lawlor, B., Gill, M., Brayne, C., Rubinsztein, D.C., Owen, M.J., Williams, J., Lovestone, S., Powell, J.F., 2012. Association of serotonin and dopamine gene pathways with behavioral subphenotypes in dementia. Neurobiol. 
Aging 33, 791-803.

Prokšelj, T., Jerin, A., Muck-Seler, D., Kogoj, A., 2014. Decreased platelet serotonin concentration in Alzheimer's disease with involuntary emotional expression disorder. Neurosci. Lett. 578, 71-74.

Reeves, S., Brown, R., Howard, R., Grasby, P., 2009. Increased striatal dopamine (D2/D3) receptor availability and delusions in Alzheimer disease. Neurology 72 , 528-534.

Rizzi, L., Rosset, I., Roriz-Cruz, M., 2014. Global epidemiology of dementia: Alzheimer's and vascular types. Biomed Res. Int. 2014, 908915.

Rosenberg, P.B., Nowrangi, M.A., Lyketsos, C.G., 2015. Neuropsychiatric symptoms in Alzheimer's disease: What might be associated brain circuits? Mol. Aspects Med. 43-44, 25-37.

Rudolph, U., Möhler, H., 2004. Analysis of GABAA receptor function and dissection of the pharmacology of benzodiazepines and general anesthetics through mouse genetics. Annu. Rev. Pharmacol. Toxicol. 44, 475-498.

Russo-Neustadt, A., Cotman, C.W., 1997. Adrenergic receptors in Alzheimer's disease brain: selective increases in the cerebella of aggressive patients. J. Neurosci. 17, 5573-5580.

Samuels, E.R., Szabadi, E., 2008. Functional neuroanatomy of the noradrenergic locus coeruleus: its roles in the regulation of arousal and autonomic function part I: principles of functional organisation. Curr. Neuropharmacol. 6, 235-253.

Sato, N., Ueki, A., Ueno, H., Shinjo, H., Morita, Y., 2009. Dopamine D3 receptor gene polymorphism influences on behavioral and psychological symptoms of dementia (BPSD) in mild dementia of Alzheimer's type. J. Alzheimers. Dis. 17, 441-448.

Schneider, L.S., Dagerman, K., Insel, P.S., 2006. Efficacy and adverse effects of atypical antipsychotics for dementia: meta-analysis of randomized, placebocontrolled trials. Am. J. Geriatr. Psychiatry 14, 191-210.

Schneider, L.S., Severson, J.A., Chui, H.C., Pollock, V.E., Bruce Sloane, R., Fredrickson, E.R., 1988. Platelet tritiated imipramine binding and MAO activity in Alzheimer's disease patients with agitation and delusions. Psychiatry Res. 25, 311322.

Seo, D., Patrick, C.J., Kennealy, P.J., 2008. Role of Serotonin and Dopamine System Interactions in the Neurobiology of Impulsive Aggression and its Comorbidity with other Clinical Disorders. Aggress. Violent Behav. 13, 383-395.

Sharp, S.I., Ballard, C.G., Chen, C.P.L.-H., Francis, P.T., 2007. Aggressive behavior and neuroleptic medication are associated with increased number of alpha1adrenoceptors in patients with Alzheimer disease. Am. J. Geriatr. Psychiatry 15, 435-437.

Silvestri, S., Seeman, M.V., Negrete, J.C., Houle, S., Shammi, C.M., Remington, G.J., Kapur, S., Zipursky, R.B., Wilson, A.A., Christensen, B.K., Seeman, P., 2000. Increased dopamine D2 receptor binding after long-term treatment with antipsychotics in humans: a clinical PET study. Psychopharmacology 152, 174180.

Sukonick, D.L., Pollock, B.G., Sweet, R.A., Mulsant, B.H., Rosen, J., Klunk, W.E., Kastango, K.B., DeKosky, S.T., Ferrell, R.E., 2001. The 5-HTTPR ${ }^{\star} S /{ }^{*} \mathrm{~L}$ polymorphism and aggressive behavior in Alzheimer disease. Arch. Neurol. 58, 
$1425-1428$.

Sultzer, D.L., Melrose, R.J., Riskin-Jones, H., Narvaez, T.A., Veliz, J., Ando, T.K., Juarez, K.O., Harwood, D.G., Brody, A.L., Mandelkern, M.A., 2017. Cholinergic Receptor Binding in Alzheimer Disease and Healthy Aging: Assessment In Vivo with Positron Emission Tomography Imaging. Am. J. Geriatr. Psychiatry 25, 342353.

Sweet, R.A., Nimgaonkar, V.L., Kamboh, M.I., Lopez, O.L., Zhang, F., DeKosky, S.T., 1998. Dopamine receptor genetic variation, psychosis, and aggression in Alzheimer disease. Arch. Neurol. 55, 1335-1340.

Sweet, R.A., Pollock, B.G., Mulsant, B.H., Rosen, J., Lo, K.H., Yao, J.K., Henteleff, R.A., Mazumdar, S., 1997. Association of plasma homovanillic acid with behavioral symptoms in patients diagnosed with dementia: a preliminary report. Biol. Psychiatry 42, 1016-1023.

Sweet, R.A., Pollock, B.G., Sukonick, D.L., Mulsant, B.H., Rosen, J., Klunk, W.E., Kastango, K.B., DeKosky, S.T., Ferrell, R.E., 2001. The 5-HTTPR polymorphism confers liability to a combined phenotype of psychotic and aggressive behavior in Alzheimer disease. Int. Psychogeriatr. 13, 401-409.

Tariot, P.N., Erb, R., Podgorski, C.A., Cox, C., Patel, S., Jakimovich, L., Irvine, C., 1998. Efficacy and tolerability of carbamazepine for agitation and aggression in dementia. Am. J. Psychiatry 155, 54-61.

Theofilas, P., Ehrenberg, A.J., Dunlop, S., Di Lorenzo Alho, A.T., Nguy, A., Leite, R.E.P., Rodriguez, R.D., Mejia, M.B., Suemoto, C.K., Ferretti-Rebustini, R.E.D.L., Polichiso, L., Nascimento, C.F., Seeley, W.W., Nitrini, R., Pasqualucci, C.A., Jacob Filho, W., Rueb, U., Neuhaus, J., Heinsen, H., Grinberg, L.T., 2016. Locus coeruleus volume and cell population changes during Alzheimer's disease progression: A stereological study in human postmortem brains with potential implication for early-stage biomarker discovery. Alzheimers. Dement. https://doi.org/10.1016/j.jalz.2016.06.2362

Thompson, A.J., Lummis, S.C.R., 2007. The 5-HT3 receptor as a therapeutic target. Expert Opin. Ther. Targets 11, 527-540.

Ueki, A., Ueno, H., Sato, N., Shinjo, H., Morita, Y., 2007. Serotonin transporter gene polymorphism and BPSD in mild Alzheimer's disease. J. Alzheimers. Dis. 12, 245253.

van der Linde, R.M., Dening, T., Stephan, B.C.M., Prina, A.M., Evans, E., Brayne, C., 2016. Longitudinal course of behavioural and psychological symptoms of dementia: systematic review. Br. J. Psychiatry 209, 366-377.

Vermeiren, Y., Janssens, J., Aerts, T., Martin, J.-J., Sieben, A., Van Dam, D., De Deyn, P.P., 2016. Brain Serotonergic and Noradrenergic Deficiencies in Behavioral Variant Frontotemporal Dementia Compared to Early-Onset Alzheimer?s Disease. J. Alzheimers. Dis. 53, 1079-1096.

Vermeiren, Y., Van Dam, D., Aerts, T., Engelborghs, S., De Deyn, P.P., 2014a. Brain region-specific monoaminergic correlates of neuropsychiatric symptoms in Alzheimer's disease. J. Alzheimers. Dis. 41, 819-833.

Vermeiren, Y., Van Dam, D., Aerts, T., Engelborghs, S., De Deyn, P.P., 2014b. Monoaminergic neurotransmitter alterations in postmortem brain regions of depressed and aggressive patients with Alzheimer's disease. Neurobiol. Aging 35, 
2691-2700.

Wang, L.Y., Shofer, J.B., Rohde, K., Hart, K.L., Hoff, D.J., McFall, Y.H., Raskind, M.A., Peskind, E.R., 2009. Prazosin for the treatment of behavioral symptoms in patients with Alzheimer disease with agitation and aggression. Am. J. Geriatr. Psychiatry $17,744-751$.

Whitford, G.M., 1986. Alzheimer's disease and serotonin: a review. Neuropsychobiology 15, 133-142.

Witte, A.V., Flöel, A., Stein, P., Savli, M., Mien, L.-K., Wadsak, W., Spindelegger, C., Moser, U., Fink, M., Hahn, A., Mitterhauser, M., Kletter, K., Kasper, S., Lanzenberger, R., 2009. Aggression is related to frontal serotonin-1A receptor distribution as revealed by PET in healthy subjects. Hum. Brain Mapp. 30, 25582570.

Wonnacott, S., 1997. Presynaptic nicotinic ACh receptors. Trends Neurosci. 20, 92-98.

Woodward, M.R., Harper, D.G., Stolyar, A., Forester, B.P., Ellison, J.M., 2014.

Dronabinol for the treatment of agitation and aggressive behavior in acutely hospitalized severely demented patients with noncognitive behavioral symptoms. Am. J. Geriatr. Psychiatry 22, 415-419.

Woolley, M.L., Marsden, C.A., Fone, K.C.F., 2004. 5-ht6 receptors. Curr. Drug Targets CNS Neurol. Disord. 3, 59-79. 